\title{
Approximate solutions of damped nonlinear vibrating system with varying coefficients under some conditions
}

\author{
Pinakee Dey ${ }^{*}$, Babul Hossain, Musa Miah, Mohammad Mokaddes Ali \\ Department of Mathematics, Mawlana Bhashani Science and Technology University, Santosh, Tangail-1902, Bangladesh.
}

Email address:

pinakee_68@yahoo.com (P. Dey)

\section{To cite this article:}

Pinakee Dey, Babul Hossain, Musa Miah, Mohammad Mokaddes Ali. Approximate solutionsof Damped Nonlinear Vibrating System with Varying Coefficients under Some Conditions. Applied and Computational Mathematics. Vol. 1, No. 1, 2012, pp. 1-5. doi: 10.11648/j.acm.20120101.11

\begin{abstract}
Krylov-Bogoliubov-Mitropolskii (KBM) method has been extended to certain damped-oscillatory nonlinear systems with varying coefficients. The solution obtained for different initial conditions for a second order nonlinear system show a good coincidence with those obtained by numerical method. The method is illustrated by an example.
\end{abstract}

Keywards: Nonlinear System, Varying Coefficient, Unperturbed Equation, Damped Oscillatory System

\section{Introduction}

Krylov and Bogoliubov [1] developed a perturbation method to obtain an approximate solution of a second order nonlinear differential system described by

$$
\ddot{x}+\omega_{0}^{2} x=-\varepsilon f(x, \dot{x})
$$

where the over dots denote the differentiation with respect to $t, \omega_{0}$ is a positive constant and $\varepsilon$ is a small parameter. Then the method was amplified and justified by Bogoliubov and Mitropolskii [2]. Mitropolskii [3] has extended the method to nonlinear differential system with slowly varying coefficients

$$
\ddot{x}+\omega_{0}^{2}(\tau) \cdot x=-\varepsilon f(x, \dot{x}, \tau), \tau=\varepsilon t
$$

Following the extended Krylov-BogoliubovMitropolskii (KBM) [1-4] method, Bojadziev and Edwards [5] studied some damped oscillatory and non-oscillatory systems modeled by

$$
\ddot{x}+c(\tau) \cdot \dot{x}+\omega^{2}(\tau) \cdot x=-\varepsilon f(x, \dot{x}, \tau),
$$

where $c(\tau)$ and $\omega(\tau)$ are positive. Murty [6] has presented a unified KBM method for both under-damped and over-damped system with constant coefficients. Shamsul [7] has presented a unified method for solving an $n$-th order differential equation (autonomous) characterized by oscillatory, damped oscillatory and non-oscillatory processes. Hung and $\mathrm{Wu}[8]$ obtained an exact solution of a differential system in terms of Bessel's functions where the coefficients varying with time in an exponential order. Recently, Roy and Shamsul [9] found an asymptotic solution of a differential system in which the coefficient changes in an exponential order of slowly varying time. In another recent article Pinakee et.al [10] has presented an extended KBM for solving nonlinear problems in which the coefficients change slowly and periodically with time. The aim of this paper is to extend the result in [10] to similar nonlinear vibrating problems in which damping forces act.

\section{Meterials and Method}

Let us consider the nonlinear differential system

$$
\begin{aligned}
& \ddot{x}+2 k(\tau) \dot{x}+\left(c_{1}+c_{2} \cos \tau+c_{3} \sin \tau\right) x= \\
& =-\varepsilon f(x, \dot{x}, \tau), \tau=\varepsilon t
\end{aligned}
$$

where the over-dots denote differentiation with respect to $t$, $\varepsilon$ is a small parameter, $c_{1}, c_{2}$ and $c_{3}$ are constants, $c_{2}=c_{3}=\mathrm{O}(\varepsilon), \tau=\varepsilon t$ is the slowly varying time, $k(\tau) \geq 0, \quad f \quad$ is a given nonlinear function. Setting $\omega^{2}(\tau)=\left(c_{1}+c_{2} \cos \tau+c_{3} \sin \tau\right), \omega(\tau)$ is known as frequency. The coefficients in Eq. (4) are slowly varying in that their time derivatives are proportional to $\mathcal{E}$.

Setting $\varepsilon=0$ and $\tau=\tau_{0}=$ constant, in Eq.(4), we obtain the unperturbed solution of (4) in the form 


$$
x(t, 0)=a_{1,0} e^{\lambda_{1}\left(\tau_{0}\right) t}+a_{2,0} e^{\lambda_{2}\left(\tau_{0}\right) t}
$$

Let Eq.(4) has two eigenvalues, $\lambda_{j}\left(\tau_{0}\right), j=1,2$, where $\lambda_{j}\left(\tau_{0}\right)$ are constants, but when $\varepsilon \neq 0, \lambda_{j}(\tau)$ vary with time. When $\varepsilon \neq 0$ we seek a solution in accordance with the KBM method, of the form

$$
x(t, \varepsilon)=a_{1}(t, \tau)+a_{2}(t, \tau)+\varepsilon u_{1}\left(a_{1}, a_{2}, t, \tau\right)+\varepsilon^{2}
$$

where $a_{1}$ and $a_{2}$ satisfy the differential equations

$$
\left.\begin{array}{l}
\dot{a}_{1}=\lambda_{1}(\tau) a_{1}+\varepsilon A_{1}\left(a_{1}, a_{2}, \tau\right)+\varepsilon^{2} \\
\dot{a}_{2}=\lambda_{2}(\tau) a_{2}+\varepsilon A_{2}\left(a_{1}, a_{2}, \tau\right)+\varepsilon^{2}
\end{array}\right\}
$$

Confining our attention to the first few term $1,2, \ldots, m$ in the series expansion of (6) and (7), we evaluate functions $u_{1}, A_{1}$ and $A_{2}$ such that $a_{1}$ and $a_{2}$ appearing in (6) and (7) satisfy (4) with an accuracy of $\varepsilon^{m+1}$. In order to determine these unknown functions it was early assumed by Murty [6], Shamsul [7] that the functions $u_{1}$ exclude all fundamental terms, since these are included in the series expansion (6) at order $\varepsilon^{0}$.

Differentiating $x(t, \varepsilon)$ two times with respect to $t$, substituting for the derivatives $\ddot{x}, \dot{x}$ and $\mathrm{x}$ in the original equation (4) and equating the coefficient of $\mathcal{E}$, we obtain

$$
\begin{aligned}
& \lambda_{1}^{\prime} a_{1}+\lambda_{2}^{\prime} a_{2}-\lambda_{2} A_{1}-\lambda_{1} A_{2}+\left(\lambda_{1} a_{1} \frac{\partial}{\partial a_{1}}+\lambda_{2} a_{2} \frac{\partial}{\partial a_{2}}\right)\left(A_{1}+A_{2}\right) \\
& +\left(\lambda_{1} a_{1} \frac{\partial}{\partial a_{1}}+\lambda_{2} a_{2} \frac{\partial}{\partial a_{2}}-\lambda_{1}\right)\left(\lambda_{1} a_{1} \frac{\partial}{\partial a_{1}}+\lambda_{2} a_{2} \frac{\partial}{\partial a_{2}}-\lambda_{2}\right) u_{1}= \\
& =-f^{(0)}\left(a_{1}, a_{2}, \tau\right),
\end{aligned}
$$

where $\lambda_{1}^{\prime}=\frac{d \lambda_{1}}{d \tau}, \lambda_{2}^{\prime}=\frac{d \lambda_{2}}{d \tau}, f^{(0)}=f\left(x_{0}, \dot{x}_{0}, \tau\right)$ and $x_{0}=a_{1}(t, \tau)+a_{2}(t, \tau)$.

It is assumed that both $f^{(0)}$ can be expanded in Taylor's series [6-7]

$$
f^{(0)}=\sum_{r_{1}, r_{2}=0}^{\infty} F_{r_{1}, r_{2}}(\tau) a_{1}^{r_{1}} a_{2}^{r_{2}}
$$

KBM [1-4], Murty [6], Shamsul [7] imposed the condition that $u_{1}$ does not contains the fundamental terms (the solution (5) is called generating solution of (4) and its terms are called fundamental terms) of $f^{(0)}$. Therefore, equation (8) can be separated into three equations for unknown functions $u_{1}$ and $A_{1}, A_{2}$ (see Shamsul [7] for details). We obtain

$$
\left(\lambda_{1} a_{1} \frac{\partial}{\partial a_{1}}+\lambda_{2} a_{2} \frac{\partial}{\partial a_{2}}-\lambda_{2}\right) A_{1}+\lambda_{1}^{\prime} a_{1}=
$$

$$
\begin{aligned}
& \sum_{r_{1}=0, r_{2}=0}^{\infty, \infty} F_{r_{1}, r_{2}}\left(a_{1}^{r_{1}}, a_{2}^{r_{2}}\right), \text { if } r_{1}=r_{2}+1 \\
& \left(\lambda_{1} a_{1} \frac{\partial}{\partial a_{1}}+\lambda_{2} a_{2} \frac{\partial}{\partial a_{2}}-\lambda_{1}\right) A_{2}+\lambda_{2}^{\prime} a_{2}= \\
= & \sum_{r_{1}=0, r_{2}=0}^{\infty, \infty} F_{r_{1}, r_{2}}\left(a_{1}^{r_{1}}, a_{2}^{r_{2}}\right), \text { if } r_{2}=r_{1}+1
\end{aligned}
$$

and

$$
\begin{aligned}
& \left(\lambda_{1} a_{1} \frac{\partial}{\partial a_{1}}+\lambda_{2} a_{2} \frac{\partial}{\partial a_{2}}-\lambda_{1}\right)\left(\lambda_{1} a_{1} \frac{\partial}{\partial a_{1}}+\lambda_{2} a_{2} \frac{\partial}{\partial a_{2}}-\lambda_{2}\right) u_{1}= \\
& \sum_{r_{1}=0, r_{2}=0}^{\infty, \infty} F_{r_{1}, r_{2}}\left(a_{1}^{r_{1}}, a_{2}^{r_{2}}\right),
\end{aligned}
$$

where $\sum_{r_{1}=0, r_{2}=0}^{\infty, \infty} F_{r_{1}, r_{2}}\left(a_{1}^{r_{1}}, a_{2}^{r_{2}}\right)$ exclude those terms for $r_{1}=r_{2} \pm 1$.

Thus the particular solutions of (10),(11) and (12) give the values of the unknown functions $A_{1}, A_{2}$ and $u_{1}$ which completes the determination of the solution of non-linear problem (4).

Example: We consider a second order nonlinear system with slowly varying coefficients

$$
\ddot{x}+2 k(\tau) \dot{x}+\left(c_{1}^{2}+c_{2} \cos \tau+c_{3} \sin \tau\right) x=-\varepsilon x^{3}
$$

Here over dots denote differentiation with respect to $t$. $c_{1}, c_{2}$ and $c_{3}$ are constants, $c_{2}=c_{3}=\mathrm{O}(\varepsilon)$, $x_{0}=a_{1}+a_{2}$ and the function $f^{(0)}$ becomes,

$$
f^{(0)}=-\left(a_{1}^{3}+3 a_{1}^{2} a_{2}+3 a_{1} a_{2}^{2}+a_{2}^{3}\right) .
$$

Following the assumption (discussed in section 2) $u_{1}$ excludes the terms $3 a_{1}^{2} a_{2}$ and $3 a_{1} a_{2}^{2}$.

We substitute in (8) and separate it into two parts as

$$
\begin{aligned}
& \lambda_{1}^{\prime} a_{1}+\lambda_{2}^{\prime} a_{2}-\lambda_{2} A_{1}-\lambda_{1} A_{2}+\left(\lambda_{1} a_{1} \frac{\partial}{\partial a_{1}}+\lambda_{2} a_{2} \frac{\partial}{\partial a_{2}}\right)\left(A_{1}+A_{2}\right)= \\
& =-\left(3 a_{1}^{2} a_{2}+3 a_{1} a_{2}^{2}\right)
\end{aligned}
$$

and

$$
\begin{aligned}
& \left(\lambda_{1} a_{1} \frac{\partial}{\partial a_{1}}+\lambda_{2} a_{2} \frac{\partial}{\partial a_{2}}-\lambda_{1}\right)\left(\lambda_{1} a_{1} \frac{\partial}{\partial a_{1}}+\lambda_{2} a_{2} \frac{\partial}{\partial a_{2}}-\lambda_{2}\right) u_{1}= \\
& =-\left(a_{1}^{3}+a_{2}^{3}\right)
\end{aligned}
$$

The particular solution of (16) is 


$$
u_{1}=-\frac{a_{1}^{3}}{2 \lambda_{1}\left(3 \lambda_{1}-\lambda_{2}\right)}-\frac{a_{2}^{3}}{2 \lambda_{2}\left(3 \lambda_{2}-\lambda_{1}\right)}
$$

Now we have to solve (15) for two functions $A_{1}$ and $A_{2}$. According with the unified KBM method $A_{1}$ contains the term $3 a_{1}^{2} a_{2}$ and $A_{2}$ contains the term $3 a_{1} a_{2}^{2}$ (Shamsul [7]) and thus we obtain the following equations

$$
\left(\lambda_{1} a_{1} \frac{\partial}{\partial a_{1}}+\lambda_{2} a_{2} \frac{\partial}{\partial a_{2}}-\lambda_{2}\right) A_{1}+\lambda_{1}^{\prime} a_{1}=-3 a_{1}^{2} a_{2}
$$

and

$$
\left(\lambda_{1} a_{1} \frac{\partial}{\partial a_{1}}+\lambda_{2} a_{2} \frac{\partial}{\partial a_{2}}-\lambda_{1}\right) A_{2}+\lambda_{2}^{\prime} a_{2}=-3 a_{1} a_{2}^{2}
$$

The particular solutions of (18) and (19) are

$$
A_{1}=-\frac{\lambda_{1}^{\prime} a_{1}}{\lambda_{1}-\lambda_{2}}-\frac{3 a_{1}^{2} a_{2}}{2 \lambda_{1}}
$$

and

$$
A_{2}=\frac{\lambda_{2}^{\prime} a_{2}}{\lambda_{1}-\lambda_{2}}-\frac{3 a_{1} a_{2}^{2}}{2 \lambda_{2}}
$$

Substituting the functional values of $A_{1}, A_{2}$ from (20) and (21) into (7) and rearranging, we obtain

$$
\dot{a}_{1}=\lambda_{1} a_{1}+\varepsilon\left(-\frac{\lambda_{1}^{\prime} a_{1}}{\lambda_{1}-\lambda_{2}}-\frac{3 a_{1}^{2} a_{2}}{2 \lambda_{1}}\right)
$$

and

$$
\dot{a}_{2}=\lambda_{2} a_{2}+\varepsilon\left(\frac{\lambda_{2}^{\prime} a_{2}}{\lambda_{1}-\lambda_{2}}-\frac{3 a_{1} a_{2}^{2}}{2 \lambda_{2}}\right)
$$

Under the transformations, $a_{1}=a e^{i \varphi} / 2$ and $a_{2}=a e^{-i \varphi} / 2$ together with $\lambda_{1}=-k+i \omega, \lambda_{2}=-k-i \omega$ equations (22) and (23) reduce to

$$
\dot{a}=\varepsilon \widetilde{A}_{1}(a)+\varepsilon^{2} \ldots
$$

and

$$
\dot{\varphi}=\omega+\varepsilon \widetilde{B}_{1}(a)+\varepsilon^{2}
$$

We shall obtain the variational equations of $a$ and $\varphi$ in the real form ( $a$ and $\varphi$ are know as amplitude and phase respectively) which transform (24) to

$$
\dot{a}=-k a-\frac{\varepsilon a \omega^{\prime}}{2 \omega}+\frac{3 \varepsilon a^{3} k}{8\left(k^{2}+\omega^{2}\right)}
$$

and

$$
\dot{\varphi}=\omega-\frac{\varepsilon k^{\prime}}{2 \omega}+\frac{3 \varepsilon a^{2} \omega}{8\left(k^{2}+\omega^{2}\right)}
$$

where $\omega=\sqrt{c_{1}+c_{2} \cos \tau+c_{3} \sin \tau}$

The variational equations (25) and (26) are in the form of the KBM [1-4] solution. The variational equations for amplitude and phase are usually appeared in a set of first order differential equations and solved by the numerical technique (see Shamsul [7]).

Therefore, the first order solution of the equation (13) is

$$
x(t, \varepsilon)=a \cos \varphi+\varepsilon u_{1}
$$

where $a$ and $\varphi$ are the solution of the equation (25) and (26) respectively.

\section{Results and Discussions}

Based on the extended KBM [1-4] method an asymptotic solution of second order damped nonlinear systems has been found in this article. In order to test the accuracy of an approximate solution obtained by a certain perturbation method, one compares the approximate solution to the numerical solution (considered to be exact). With regard to such a comparison concerning the presented KBM method of this article, we refer to the works of Murty [6], and Shamsul [7]. In our present paper, for different initial conditions, we have compared the perturbation solutions (27) of Duffing's equations (13) to those obtained by Runge-Kutta Fourth-order procedure.

First of all, for damping force $-.002 \sqrt{\cos \tau}$, i.e., $k=.001 \sqrt{\cos \tau}, x$ is calculated by (13) with initial conditions $\quad[x(0)=1.00000, \dot{x}(0)=0.00000] \quad$ or $a=1.000001, \varphi=-.001161 \quad, \quad \varepsilon=.1 \quad$ and $\omega=\omega_{0} \sqrt{\left(c_{1}+c_{2} \cos \tau+c_{3} \sin \tau\right)}$. Then corresponding numerical solutions is also computed by Runge-Kutta fourth-order method. All the results are shown in Fig.1. From Fig. 1 it is clear that the asymptotic solution (27) shows a good coincidence with the numerical solution of equation (13). We have find the approximate solutions of the same problem for damping force $-.02 \sqrt{\cos \tau}$, i.e., $k=.01 \sqrt{\cos \tau} \quad$ with initial conditions $[x(0)=1.00000, \dot{x}(0)=0.00000] \quad$ or $a=1.000043, \varphi=-.009277 \quad, \quad \varepsilon=.1 \quad$ and $\omega=\omega_{0} \sqrt{\left(c_{1}+c_{2} \cos \tau+c_{3} \sin \tau\right)}$, for damping force $-.1 \sqrt{\cos \tau}$, i.e., $k=.05 \sqrt{\cos \tau}$ with initial conditions $[x(0)=1.00000, \dot{x}(0)=0.00000] \quad$ or $a=1.001075, \varphi=-.046354 \quad$ for $\quad \varepsilon=.1 \quad$ and $\omega=\omega_{0} \sqrt{\left.c_{1}+c_{2} \cos \tau+c_{3} \sin \tau\right)}$ and for damping force $-.2 \sqrt{\cos \tau}$, i.e., $k=.1 \sqrt{\cos \tau}$ with intial conditions 
$[x(0)=1.00000, \dot{x}(0)=0.00000]$

$a=1.004295, \varphi=-.092516$ for $\varepsilon=.1 \quad$ and $\omega=\omega_{0} \sqrt{\left.c_{1}+c_{2} \cos \tau+c_{3} \sin \tau\right)}$. The corresponding numerical solutions have also been computed by RungeKutta fourth-order method. From Fig. 2 ,Fig. 3 and Fig. 4 we observe that the approximate solutions agree with numerical results nicely.

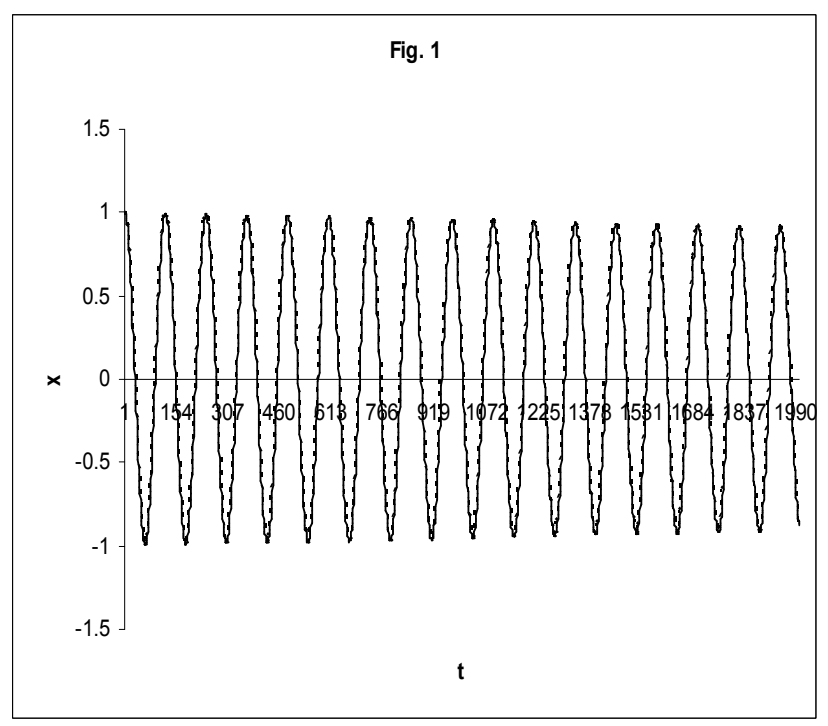

Fig. 1. Perturbation solution (dotted line) with corresponding numerical solution (solid line) are plotted with initial conditions $a=1.000001, \varphi=-.001161$ $[x(0)=1.00000, \dot{x}(0)=0.00000]$ for $e=.1, h=.05$., with damping coefficient is $k=.001 \sqrt{\cos \tau}$

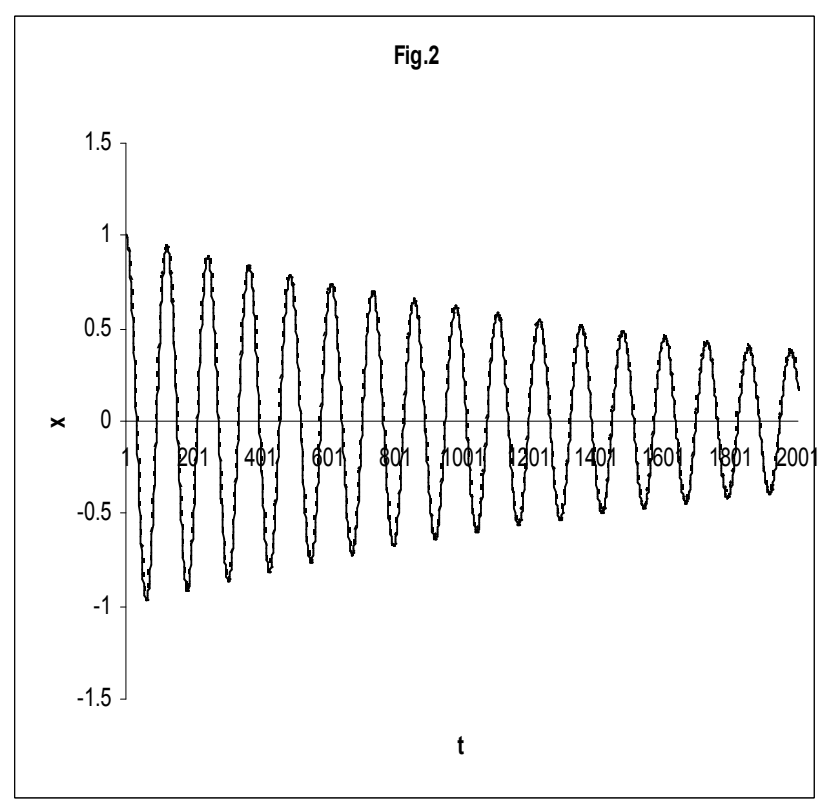

Fig. 2. Perturbation solution (dotted line) with corresponding numerical solution (solid line) are plotted with initial conditions $a=1.000045, \varphi=-.009475$ $[x(0)=1.00000, \dot{x}(0)=0.00000]$ for $e=.1, h=.05$., with damping coefficient is $k=.01 \sqrt{\cos \tau}$

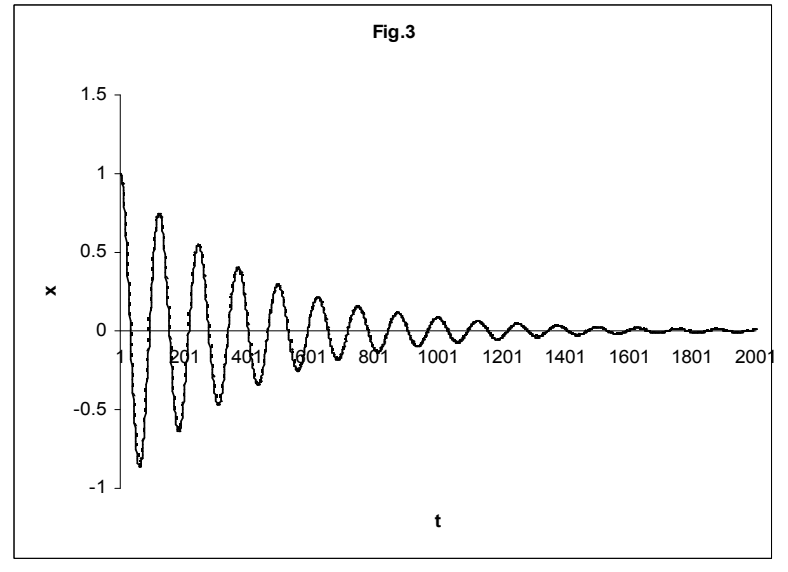

Fig. 3. Perturbation solution (dotted line) with corresponding numerical solution (solid line) are plotted with initial conditions $a=1.001077, \varphi=-.046395$

$[x(0)=1.00000, \dot{x}(0)=0.00000]$ for $e=.1, h=.05$, with damping coefficient is $k=.05 \sqrt{\cos \tau}$

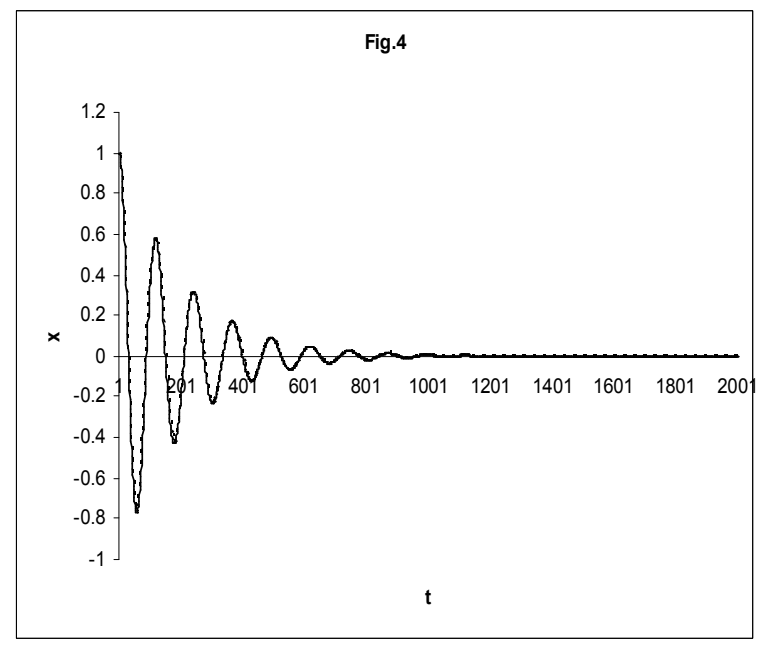

Fig. 4. Perturbation solution (dotted line) with corresponding numerical solution (solid line) are plotted with initial conditions $a=1.004281, \varphi=-.092362$

$[x(0)=1.00000, \dot{x}(0)=0.00000]$ for $e=.1, h=.05$, with damping coefficient is $k=.1 \sqrt{\cos \tau}$.

\section{Conclusion}

The KBM method is extended and used to obtain asymptotic solutions of the nonlinear vibrating systems under the action of several damping forces, where the coefficients change slowly with time. The method is a generalization of the asymptotic KBM method. The derivation of the present formula is quite different from the classical KBM method. A direct attempt to obtain the general formulae is a tremendously-difficult task, which is not practical. Where as the present method is systematic and easy to understand. Therefore, the formulation, as well as the determination, of the solution is much simpler than that of KBM technique in both damped and un-damped cases. 


\section{Acknowledgement}

Thanks to reviewers for their comments and helpful suggestions to. The authors are also grateful to Prof. Dr. M. Nurul Islam (V.C Mawlana Bhashani Science and Technology University) for several time discussions with him.

\section{References}

[1] N.N, Krylov and N.N., Bogoliubov, Introduction to Nonlinear Mechanics. Princeton University Press, New Jersey, 1947.

[2] N. N, Bogoliubov and Yu. Mitropolskii, Asymptotic Methods in the Theory of nonlinear Oscillations, Gordan and Breach, New York, 1961.

[3] Yu.,Mitropolskii, "Problems on Asymptotic Methods of Non-stationary Oscillations" (in Russian), Izdat, Nauka, Moscow, 1964.

[4] I. P. Popov, "A generalization of the Bogoliubov asymptotic method in the theory of nonlinear oscillations", Dokl.Akad. Nauk SSSR 111, 1956, 308-310 (in Russian).
[5] G.,Bojadziev, and J. Edwards, "On Some Asymptotic Methods for Non-oscillatory and Oscillatory Processes", Nonlinear Vibration Problems, 20, 1981, pp69-79.

[6] I.S.N. Murty, "A Unified Krylov-Bogoliubov Method for Second Order Nonlinear Systems", Int. J. nonlinear Mech., 6, 1971, pp45-53.

[7] M.,Shamsul Alam, "Unified Krylov-BogoliubovMitropolskii Method for Solving n-th Order Nonlinear System with Slowly Varying Coefficients", Journal of Sound and Vibration, 256, 2003, pp987-1002.

[8] Hung Cheng and Tai Tsun Wu, "An Aging Spring, Studies in Applied Mathematics", 49, 1970, pp183-185.

[9] K.C, Roy, M. Shamsul Alam, "Effects of Higher Approximation of Krylov- Bogoliubov-Mitropolskii Solution and Matched Asymptotic Solution of a Differential System with Slowly Varying Coefficients and Damping Near to a Turning Point", Vietnam Journal of Mechanics, VAST, 26, 2004, pp182-192.

[10] Pinakee Dey., Harun or Rashid, M. Abul Kalam Azad and M S Uddin, "Approximate Solution of Second Order Time Dependent Nonlinear Vibrating Systems with Slowly Varying Coefficients", Bull. Cal. Math. Soc, 103, (5), 2011, pp371-38. 